\title{
Aggregate dust connections and emissivity enhancements ${ }^{\star}$
}

\author{
M. Köhler ${ }^{1}$, V. Guillet ${ }^{1}$, and A. Jones ${ }^{1}$ \\ Institute d'Astrophysique Spatiale, Universite Paris Sud, 91405 Orsay, France \\ e-mail: mkoehler@ias.u-psud.fr
}

Received 21 December 2010 / Accepted 31 January 2011

\begin{abstract}
Context. Observations of cold condensed clouds in the interstellar medium show an enhancement in the dust emissivity at long wavelengths. Model calculations with the discrete-dipole approximation (DDA) can explain this enhancement with the coagulation of dust particles into aggregates.

Aims. We study the nature of grain-grain contacts and their effects on the aggregate optical properties.

Methods. We use DDA and the T-matrix method (TMM) to calculate the absorption properties of aggregate dust grains and analyse where and why the enhancement in the emissivity occurs.

Results. We find that the absorption coefficient changes with material composition and with the contact area between monomers. A larger contact area, with DDA, compared to a zero-point contact with TMM, results in an enhancement of the absorption coefficient for wavelengths where the considered material has a large value $n$ (the real part of the refractive index).

Conclusions. DDA seems to be the most realistic way of taking into account "real" inter-particle contact effects in aggregate particles.
\end{abstract}

Key words. dust, extinction - methods: miscellaneous

\section{Introduction}

Far-infrared (FIR) observations of dust in the interstellar medium (ISM) suggest differences in grain properties between cold condensed clouds and the diffuse ISM. The diffuse ISM component has a typical temperature of about $17.5 \mathrm{~K}$ (Boulanger et al. 1996) while the colder condensation component has a typical temperature of about $12.5 \mathrm{~K}$ (del Burgo \& Laureijs 2005). Observations with ISO, Spitzer, SPM/PRONAOS and IRAS of different regions in the interstellar medium indicate increased dust emissivities in colder regions (Bernard et al. 1999; del Burgo et al. 2003; Stepnik et al. 2003; del Burgo \& Laureijs 2005; Kiss et al. 2006; Ridderstad et al. 2006; Lehtinen et al. 2007; Bot et al. 2009; Paradis et al. 2009). This increase in emissivity observed at wavelengths $\geq 200 \mu \mathrm{m}$ is often explained by a change in the properties of the cold dust. The properties of the dust can change through the coagulation of small particles into aggregates (e.g. Bernard et al. 1999; del Burgo et al. 2003; Stepnik et al. 2003; Ridderstad et al. 2006; Paradis et al. 2009), by the accretion of mantles onto the grains (e.g. Kiss et al. 2006) or by an increase in the grain size (e.g. Henning et al. 1995).

Interplanetary dust particles (IDPs) collected in the stratosphere show, in some cases, an aggregate structure (e.g. Brownlee 1978). These aggregate IDPs consist of irregularlyshaped monomers which are on average 0.1 to $0.3 \mu \mathrm{m}$ in size (e.g. Rietmeijer 1998). Some aggregate IDPs show a very open structure while others are more smooth and compact. Aggregates are also assumed to describe cometary dust particles and dust particles in some extra-solar planetary systems, e.g., the system of $\beta$ Pictoris (Greenberg \& Li 1997; Li \& Greenberg 1998). Aggregate dust particles must also be considered in the denser ISM where coagulation has occurred.

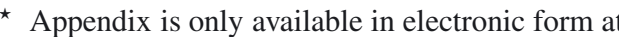
http: //www . aanda.org
}

Compared to spherical particles, extended aggregate structures show a stronger emission in the FIR (Wright 1987; Bazell \& Dwek 1990; Fogel \& Leung 1998; Stepnik et al. 2003) ${ }^{1}$ while at short wavelengths the emission of aggregate particles is similar to that of spherical grains. This effect was also observed when the absorption coefficients of hollow spheres and porous particles calculated with effective medium theories were compared to compact spherical grains (Jones 1988). This enhancement at long wavelengths leads to a decrease in grain temperatures of typically 10-20\%, compared to compact spherical particles (Fogel \& Leung 1998).

Different theories are available to calculate the optical properties of aggregates: e.g., T-matrix method (TMM) (Mackowski \& Mishchenko 1996), generalized multi-particle Mie theory (GMM) (Xu 1995) and discrete-dipole approximation (DDA) (Purcell \& Pennypacker 1973; Draine 1988; Draine \& Flatau 2010). All of these theories can be used to determine the scattering and absorption properties of aggregates. Using DDA the particle is divided into interacting dipoles which results in a discretised description of the particle's surface. The more dipoles that are used to describe the particle the more accurate the description of the surface and therefore the result, but then more computer memory and CPU time are needed for the calculations. GMM and TMM codes are only available for selected particle shapes. DDA can treat particles of arbitrary shape but is limited to particles with size parameter $x=2 \pi a_{\mathrm{V}} / \lambda \lesssim 25$, where $a_{\mathrm{V}}$ is the radius of a volume-equivalent sphere and $\lambda$ is the wavelength, and to materials with optical constants $|m-1| \lesssim 2$, where $m=n+\mathrm{i} k$ (Draine \& Flatau 2010).

Calculations of the absorption coefficient $Q_{\text {abs }}$ of single spherical particles with DDA show good agreement with Mie

1 The value of $<5 \%$ determined for grains of amorphous carbon by Bazell \& Dwek (1990) was corrected by Rouleau \& Martin (1993) to $<30 \%$. 
theory (Mie 1908; Bohren \& Huffman 1983) over a wide wavelength range up to $2 \mathrm{~mm}$ (e.g. Draine 1988). However, small deviations appear due to surface properties (e.g. Draine 1988). Compared to spherical particles, aggregates of the same mass show extinction enhancements of 1.2-1.3 at mm wavelengths for silicate and enhancements that can vary between 1.4-1.6 for carbon, for calculations with DDA and grain sizes in the Rayleigh regime (e.g. Stognienko et al. 1995). These authors concluded that the extinction depends on the refractive index of the material and on the topology of the particle. Compared to the results for aggregates modelled with DDA, the results with GMM are smaller and the deviations increase with increasing refractive index (Michel et al. 1996).

In this study we investigate the following question: What is the origin for the enhancement in $Q_{\mathrm{abs}}$ found in DDA calculations compared to TMM calculations for the same aggregate structure and same refractive indices? In Sect. 2 we describe our model to calculate $Q_{\text {abs }}$ for aggregates and present the results in Sect. 3. We finish the paper with a discussion (Sect. 4) and concluding remarks (Sect. 5).

\section{Model}

We carry out model calculation with TMM and DDA for aggregate dust particles and determine their absorption coefficient $Q_{\mathrm{abs}}$. The main difference between TMM and DDA is the shape of the monomers forming the aggregate. While for TMM the monomers are perfectly spherical, the surface of the DDA monomers can deviate significantly from perfect spheres, depending on the number of dipoles. This results in different connections between monomers when they are in aggregates. In the case of TMM the connection is a point contact whereas in the case of DDA the connection is always an area equivalent to at least one dipole. For a good result the monomer should be built with as many dipoles as possible, then the contact between the monomers will be even larger than one dipole. For DDA calculations we therefore have to chose an appropriate number of dipoles per monomer in order to describe the contact area in the aggregates. We investigate how the connection between monomers influences the absorption efficiency of an aggregate and how this affects the optical constants.

We consider aggregates of equal-sized monomers. Note that we do not mix monomer compositions and therefore our aggregates are composed of either pure silicate or pure carbon monomers.

\subsection{Building aggregates}

The aggregates are constructed such that the monomers are located on a cubic grid. This allows us to control the contact area between two monomers in detail for DDA and to ensure that the contact area between any two monomers is the same. For all calculations we consider compact spherical monomers. We choose two different materials: astronomical silicate (Draine \& Lee 1984) and amorphous carbon (AC1) (Rouleau \& Matin 1991). We define $\varnothing_{N}^{C}$ where $N$ is the number of dipoles across the diameter of the monomer and $C$ is the number of dipoles across the contact area between two monomers, shown here for $N=13$ and $C=5$ :

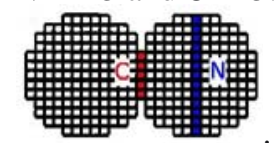

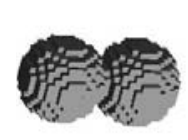
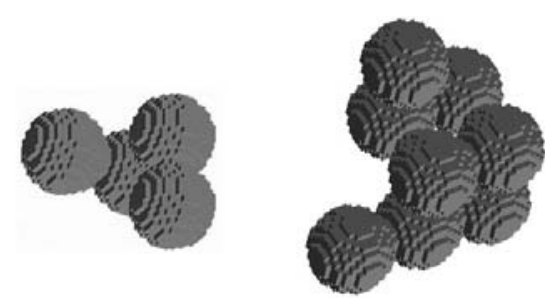

Fig. 1. Aggregates consisting of 2, 4, 8 and 16 monomers built of dipoles. Each dipoles is indicated by a cube.

Aggregates are characterised by their fractal dimension $D_{\mathrm{f}}$. In our calculations we assume $D_{\mathrm{f}}=2\left(D_{\mathrm{f}}=3\right.$ for compact aggregates, $D_{\mathrm{f}}=1$ for linear aggregates, and $1<D_{\mathrm{f}}<3$ for fractal aggregates). For a fractal aggregate, within a radius $r$, the number of primary particles $n$ of radius $a$ is given by (e.g. Bazell \& Dwek 1990):

$n(r)=\left(\frac{r}{a}\right)^{D_{\mathrm{f}}}$

In order to build the aggregate, we start by placing one grain at the aggregate centre. Then, using the above equations, we calculate the number of sub-grains in the concentric shell between the radii $a$ and $a+2 a$. After that, we randomly place each sub-grain into the shell $(a, a+2 a)$ such that each new grain is in contact with at least one grain that is already a part of the aggregate. Finally, when the shell $(a, a+2 a)$ is full, we repeat the process using successive shell radii increased by $2 a$ until all the subgrains are placed in the required aggregate. Using this method, we build aggregates made of spherical sub-grains with a constant radius $a$ and with the required values of $n$ and $D_{\mathrm{f}}$.

The coordinates of these monomers in the aggregate are then multiplied by $N$, which is the number of dipoles of monomers across the diameter. These new coordinates are the centres of the monomers and the dipoles are placed on a cubic grid around the centre so as to build a spherical monomer.

Examples of aggregates consisting of 2, 4, 8, and 16 monomers, where each monomer is 13 dipoles in diameter $\left(\varnothing_{13}^{5}\right)$, are shown in Fig. 1 where each dipole is represented by a cube.

\subsection{Normalisation}

We calculate the absorption cross section $C_{\mathrm{abs}}=Q_{\mathrm{abs}} \pi a_{\mathrm{V}}^{2}$, where $a_{\mathrm{V}}$ is the radius of a volume equivalent sphere and $Q_{\mathrm{abs}}$ is the absorption coefficient for the aggregate. The radius of the volume equivalent sphere is $a_{\mathrm{V}}=a_{0} N^{\frac{1}{3}}$, where $a_{0}=0.1 \mu \mathrm{m}$ is the radius of a monomer and $N$ is the number of monomers in the aggregate. In all calculations we assume a constant monomer radius of $a_{0}=0.1 \mu \mathrm{m}$. For aggregates of 2, 4, 8 and 16 monomers the radii of the volume-equivalent spheres are $0.126 \mu \mathrm{m}, 0.159 \mu \mathrm{m}$, $0.200 \mu \mathrm{m}$ and $0.252 \mu \mathrm{m}$, respectively. We then divide the absorption cross section by the total volume, $V$, of the monomers in the aggregate. For all our calculations the size of the particle is much smaller than the wavelength and in this Rayleigh regime $C \propto V$.

For the normalization we divide $C_{\mathrm{abs}} / V$ by $C_{\mathrm{abs}}^{\mathrm{sph}} / V^{\mathrm{sph}}$, where $C_{\mathrm{abs}}^{\mathrm{sph}}$ is the absorption cross section of one monomer and $V^{\text {sph }}$ is the volume of one monomer with $a_{0}=0.1 \mu \mathrm{m}$. We normalise to one single monomer in order to quantify the increase for the connected monomers because for separated monomers $C_{\text {abs }} / V=$ $C_{\mathrm{abs}}^{\mathrm{sph}} / V^{\mathrm{sph}}$. 

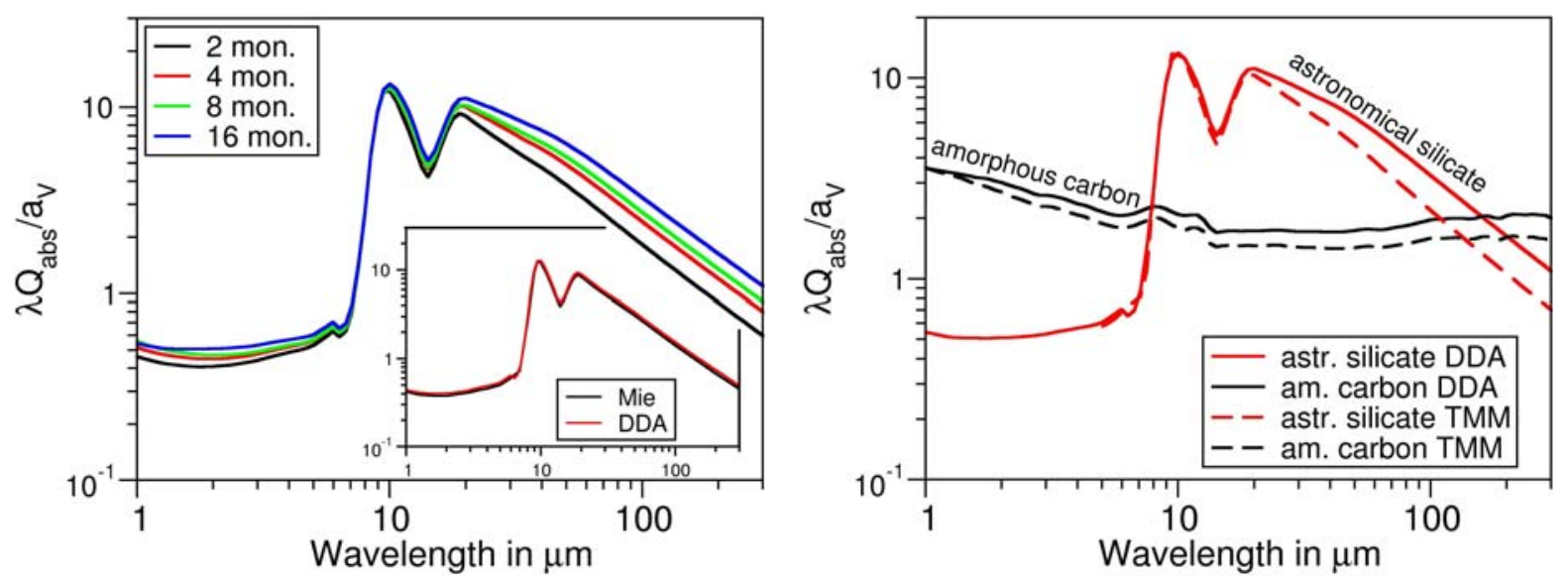

Fig. 2. Left: $\lambda Q_{\mathrm{abs}} / a_{\mathrm{V}}$ for astronomical silicate (Draine \& Lee 1984) aggregates with $2,4,8$ and 16 monomers using DDA. We assume $\emptyset_{13}^{5}$ and average over 7 orientations. The small plot shows $\lambda Q_{\mathrm{abs}} / a_{\mathrm{V}}$ for a single sphere of astronomical silicate calculated with Mie theory (black line) and DDA (red line). Right: $Q_{\mathrm{abs}} / a_{\mathrm{V}}$ versus wavelength for amorphous carbon (black) and astronomical silicate (red) aggregates of 16 monomers. The calculations are for DDA (solid lines) $\left(\varnothing_{13}^{5}\right.$, average over 7 orientations) and TMM (dashed lines).

We denote
$Q_{\mathrm{abs}}^{\text {normalised }}=\frac{C_{\mathrm{abs}} / V}{C_{\mathrm{abs}}^{\mathrm{sph}} / V^{\mathrm{sph}}}$.

\section{Results}

To analyse the enhancement in the absorption coefficient $Q_{\mathrm{abs}}$ with DDA, compared to TMM, we first calculate $Q_{\mathrm{abs}}$ for different material compositions and different aggregate sizes (by varying the number of monomers) over a wide wavelength range. We do not show results calculated with GMM since they are essentially the same results as TMM. For these calculations we assume $\emptyset_{13}^{5}$.

In Fig. 2 (left) $\lambda Q_{\mathrm{abs}} / a_{\mathrm{V}}$ is plotted versus wavelength for aggregates of $2,4,8$ and 16 monomers of astronomical silicate obtained with DDA. We note that an enhancement of $\lambda Q_{\mathrm{abs}} / a_{\mathrm{V}}$ occurs by increasing the number of monomers and that the enhancement is largest for wavelengths greater than about $\gtrsim 50 \mu \mathrm{m}$.

In Fig. 2 (right) $\lambda Q_{\mathrm{abs}} / a_{\mathrm{V}}$ is plotted versus wavelength for aggregates consisting of 16 monomers of amorphous carbon and astronomical silicate. We show results obtained with DDA (solid line) and TMM (dashed line). Depending on wavelength and material composition the values of $\lambda Q_{\mathrm{abs}} / a_{\mathrm{V}}$ are enhanced with respect to TMM at long wavelengths $(\lambda>20 \mu \mathrm{m})$. For amorphous carbon aggregates the enhancement increases with increasing wavelength over the entire wavelength range. This increase was also observed by Bazell \& Dwek (1990) who concluded that the difference is due to the dielectric properties of the material.

These calculations lead to the questions we would like to investigate in the following: 1) why do the deviations between the DDA and TMM results differ with wavelength and material composition? 2) Why is the enhancement in $Q_{\text {abs }}$ larger for DDA than for TMM? 3) Why does the enhancement increase with the number of monomers?

\subsection{Optical constants}

The enhancement in $Q_{\mathrm{abs}}$ using DDA, compared to TMM, differs with wavelength and material composition and so we now consider whether this enhancement might be due to the optical constants characteristic of each material. We now calculate $Q_{\text {abs }}$ for aggregates consisting of two monomers assuming different

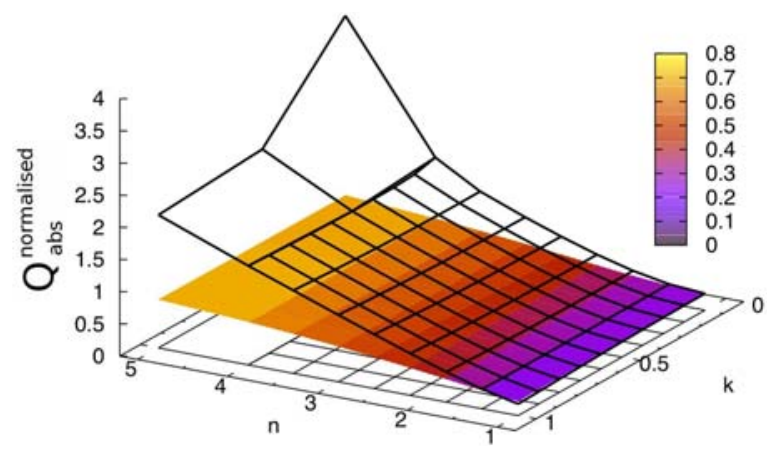

Fig. 3. $Q_{\text {abs }}^{\text {normalised }}$ versus $n$ and $k$ for aggregates consisting of 2 monomers $\left(\emptyset_{25}^{13}\right)$. The coloured surface shows the results for TMM and the black grid shows the results for DDA. At $\lambda=250 \mu \mathrm{m}$ the optical constants are $n=3.401$ and $k=0.199$ for astronomical silicate (Draine \& Lee 1984), $n=2.857$ and $k=0.282$ for amorphous carbon (AC1) (Rouleau \& Matin 1991) and $n=1.808$ and $k=0.0227$ for water ice (Warren 1984).

optical constants, namely varying $n$ from 1 to 4.5 and $k$ from 0 to 1. Additionally, we also assume $n=5.0$ for $k=0.1,0.5$ and 1.0 as an extreme case. The calculations are carried out using DDA and TMM for comparison. For DDA we consider $\emptyset_{25}^{13}$ with the results averaged over 7 orientations. We calculate $Q_{\text {abs }}^{\text {normalised }}$ where $C_{\mathrm{abs}}^{\mathrm{sph}}$ is calculated with Mie theory (Mie 1908; Bohren \& Huffman 1983).

In Fig. $3 Q_{\text {abs }}^{\text {normalised }}$ is plotted versus $n$ and $k$. The coloured surface shows the results for TMM and the black grid shows the results for DDA. For both methods we find that $Q_{\text {abs }}^{\text {normalised }}$ increases with increasing $n$ and is nearly constant with $k$. Additionally, with increasing $n$ the deviations between the results of the two methods increase. The deviations are almost independent of $k$. The results presented in Fig. 3 lead us to conclude that the absorption coefficient at long wavelengths is enhanced as $n$ increases.

In Fig. 4 the optical constants, $n$ and $k$, are plotted for astronomical silicate (Draine \& Lee 1984) and amorphous carbon (AC1) (Rouleau \& Matin 1991). For astronomical silicate $n$ is large and constant at wavelengths longward of $\sim 100 \mu \mathrm{m}$. For amorphous carbon $n$ increases with increasing wavelength. This explains why the deviations occur for astronomical silicate at 


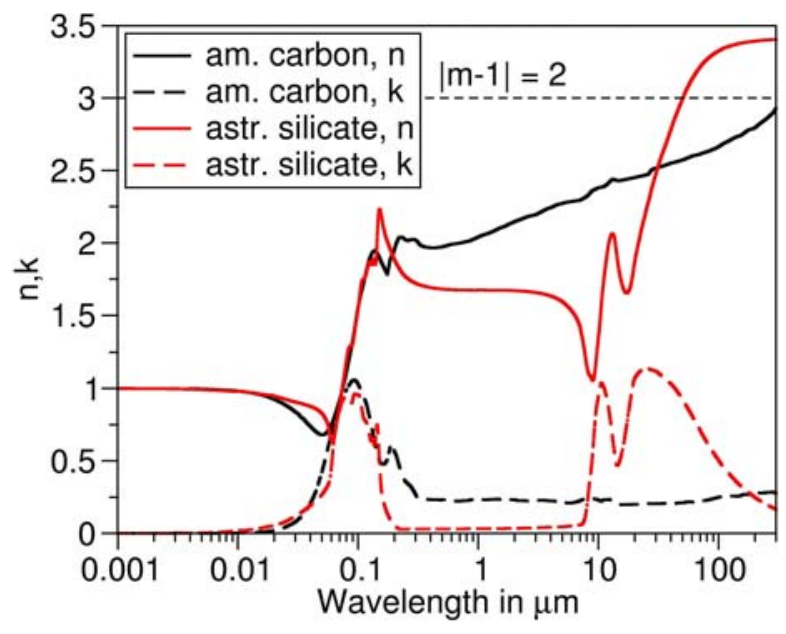

Fig. 4. The optical constants $n$ (solid line) and $k$ (dashed line) as a function of the wavelength for astronomical silicate (red curve) and amorphous carbon (black curve).

long wavelengths while the deviations increase steadily with increasing wavelength for amorphous carbon (see Fig. 2). This also explains why the enhancement for silicate saturates since $n$ is constant roughly longward of $\sim 100-200 \mu \mathrm{m}$. In Fig. 4 we also include the limit $|m-1| \leq 2$ for DDA given by Draine \& Flatau (2010). Our comparison with the TMM method shows that up to $n=3.5$ the results with DDA seem reasonable (see Fig. 3). For $n>5$ the deviations between the results of the two methods are very large, which is due to the limitations imposed by the DDA method. This was further investigated by calculating $\lambda Q_{\mathrm{abs}} / a_{\mathrm{V}}$ for a single monomer of astronomical silicate with DDA and Mie theory for comparison (see small figure in Fig. 2). Also at long wavelengths where $n=3.4$ the results with both methods are similar, small deviations occur with DDA over the entire wavelength range due to the surface structure.

We conclude that, in the case of astronomical silicate, DDA can safely be used to study enhanced particle emissivities at long wavelengths.

\subsection{Contact area}

In the following sections we investigate why the derived values of $Q_{\mathrm{abs}}$ for aggregates are larger when the calculations are carried out with DDA as compared to TMM.

\subsubsection{Distance between monomers}

To understand the influence of the connection between the monomers on $Q_{\text {abs }}$ we consider two separated spheres and move them towards each other until they connect. With decreasing distance between the monomers we calculate $Q_{\mathrm{abs}}$ using TMM and DDA. The initial distance between the monomers is the radius of one monomer. When the monomers connect this leads to a point contact in the case of TMM and in $\varnothing_{25}^{7}$ for DDA. To minimize the surface effects we assume the larger number of dipoles $(N=25)$.

Since differences in $Q_{\mathrm{abs}}$ occur with different materials we carry out calculations for aggregates consisting of astronomical silicate as well as amorphous carbon. We chose the wavelength of $250 \mu \mathrm{m}$ for the calculations in order to study in detail the reason for the observed enhancement at long wavelength seen in Fig. 2. The results with DDA are averaged over 7 orientations and the results with TMM are averaged over 180 orientations. In contrast to TMM, calculations with DDA are more time consuming and since we are only considering 2 monomers, 7 orientations are sufficient. For larger aggregates we increase the number of orientations in our calculations. We calculate $Q_{\mathrm{abs}}^{\text {normalised }}$, where $C_{\mathrm{abs}}^{\mathrm{sph}}$ is calculated with the same method as used for the aggregate.

Figure 5 shows $Q_{\text {abs }}^{\text {normalised }}$ for TMM (magenta curve) and DDA (black curve) with the differences between the results for the two methods shown as green curves. This comparison shows that, with decreasing distance between the monomers, $Q_{\text {abs }}^{\text {normalised }}$ increases for both theories (DDA and TMM). For connected monomers DDA gives a larger result than TMM. As Fig. 5 shows, the strength of this enhancement depends on the aggregate material composition: the difference between $Q_{\text {abs }}^{\text {normalised }}$ obtained with DDA and that obtained with TMM is $25 \%$ for amorphous carbon and $38 \%$ for astronomical silicate, at the considered wavelength, and for a contact of $C=7$. For separated monomers the difference in $Q_{\mathrm{abs}}^{\text {normalised }}$ between the two methods is smaller than $9 \%$ and is the result of differences in the surface structures of the particles. This difference can be decreased by assuming a larger number of dipoles per monomer.

These calculations show that the increase in $Q_{\text {abs }}^{\text {normalised }}$ calculated with DDA apparently results from the larger contact area between the monomers, as compared to the point contact between the monomers for TMM. In the following we further investigate the influence of the contact area between monomers on the absorption coefficient.

\subsubsection{Size of the contact area}

The sketches in Fig. 6 show different contact areas between two monomers (a dimer). For each monomer we assume $\varnothing_{13}^{C}$, with $C$ between 0 and 11, $C=0$ indicates that the monomers are not connected. In Fig. 6, from a) to i), the contact area between the monomers increases. This increase occurs either by merging the particles (black) or by filling the gap of 1 dipole width with dipoles (red). We distinguish between these two cases for contact areas larger than $C=3$. The possible contacts are summarized in Table 1.

In the case of merged monomers with a contact area of $C=5,7$ and 9 the contact area can be either one or two layers with the same number of dipoles across (see Fig. 7 for a contact area 5 dipoles across). We therefore calculate the absorption coefficient for both cases and take the average. For all other aggregates we consider the cases shown in Fig. 6.

Considering these different contact areas we calculate the absorption coefficient for aggregates consisting of 2, 3 and 4 monomers using DDA. The monomers are again located on a cubic grid so that the contact areas can be carefully controlled. We average over 216 orientations for each aggregate of astronomical silicate and calculate the absorption coefficient at a wavelength of $250 \mu \mathrm{m}$.

Aggregates with more than 2 monomers can have different shapes: for aggregates with 3 monomers two shape are possible, a row and an angle shape. For aggregates with 4 monomers seven different shapes are possible, shown in Fig. 8. We find that for aggregates of 3 and 4 monomers $Q_{\text {abs }}^{\text {normalised }}$ is larger the more elongated the aggregate. This theoretical result was predicted by Henning et al. (1995). In this study, we average over the possible shapes (equal-weighted) which can be formed by the monomers. Excluding the most compact and most elongated form in the case of 4 monomers leads to a deviation of $<3 \%$. 
M. Köhler et al.: Aggregate dust connections and emissivity enhancements
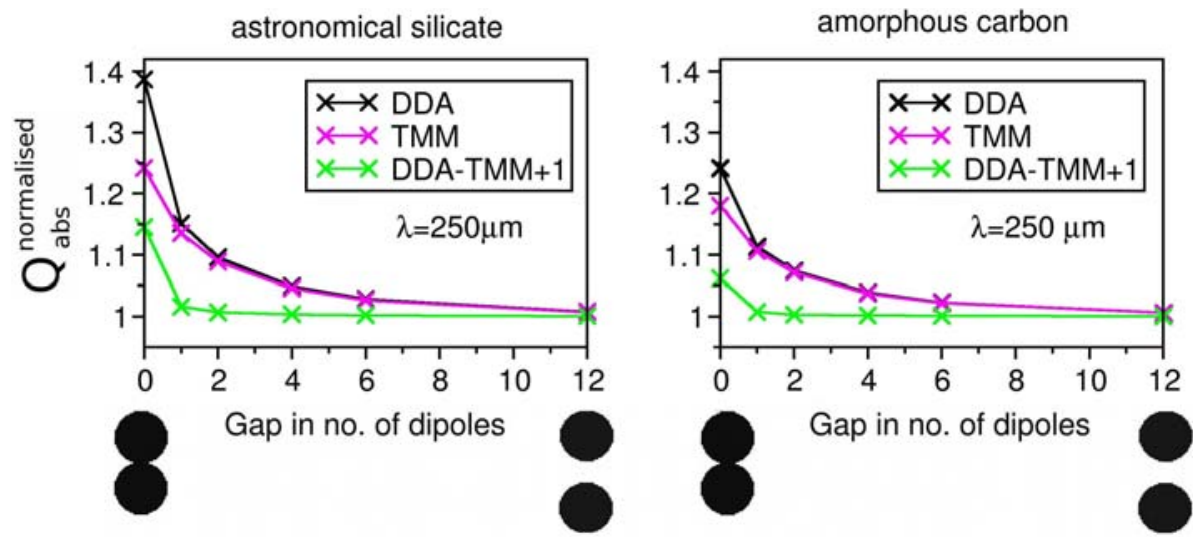

Fig. 5. $Q_{\text {abs }}^{\text {normalised }}$ versus the distance between two monomers in number of dipoles: DDA (black curve) $\left(\varnothing_{25}^{7}\right)$ and TMM (red curve). The difference plus one between the two methods is shown in green. The results are shown for dimers consisting of astronomical silicate (left) and amorphous carbon (right) at $\lambda=250 \mu \mathrm{m}$. The sketches below show the cases of largest separation and contact in the case of DDA.

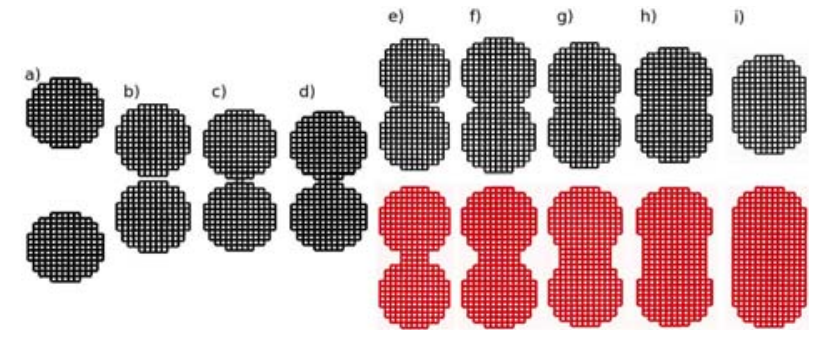

Fig. 6. Possible contact areas shown in cross-section between two monomers. Each square indicates one dipole. Each monomer is constructed of 13 dipoles in diameter $\left(\varnothing_{13}^{\mathrm{C}}\right)$. From left to right the contact area increases. a) Monomers separated by 12 dipoles $\left(\varnothing_{13}^{0}\right)$. b) Monomers separated by 1 dipole $\left(\varnothing_{13}^{0}\right)$. From c) to $\left.\mathbf{h}\right)$ the contact area increases as $\varnothing_{13}^{1}, \varnothing_{13}^{3}, \varnothing_{13}^{5}, \varnothing_{13}^{7}, \varnothing_{13}^{9}$ and $\varnothing_{13}^{11}$.

Table 1. Summary of the different contacts between two monomers with $\varnothing_{13}^{C}$.

\begin{tabular}{lccc}
\hline \hline Case & Monomer contact & $C$ & Distance \\
\hline a) & separated & 0 & 25 \\
b) & separated & 0 & 14 \\
c) & filled gap & 1 & 14 \\
d) & filled gap & 3 & 14 \\
e) red & filled gap & 5 & 14 \\
f) red & filled gap & 7 & 14 \\
g) red & filled gap & 9 & 14 \\
h) red & filled gap & 11 & 14 \\
i) red & filled gap & 13 & 14 \\
\hline e) black & merged monomers & 5 & $12 / 13$ \\
f) black & merged monomers & 7 & $12 / 13$ \\
g) black & merged monomers & 9 & $10 / 11$ \\
h) black & merged monomers & 11 & 9 \\
i) black & merged monomers & 13 & 5 \\
\hline
\end{tabular}

Notes. $C$ is given for each case; $C=0$ indicates no contact between the monomers (case a) and b)). The second column gives how the connection between the monomers is obtained. The third column gives the distance between the centres of monomers in number of dipoles. Two numbers separated by " $"$ " indicates that we calculated the average over two possible cases (see text and Fig. 7).

The results for $Q_{\text {abs }}^{\text {normalised }}$ are shown in Fig. 9. On the $x$-axis the $S 12$ tick mark indicates two monomers separated by 12 dipoles. The tick mark $S 1$ shows the case for monomers separated by 1 dipole. The following numbers on the $x$-axis

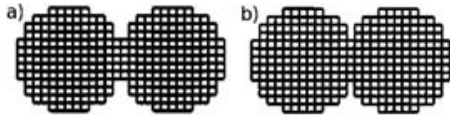

Fig. 7. The contact area can be either one or two layers with the same number of monomers. This is indicated in this sketch by a contact area of 5 dipoles across $\left(\varnothing_{13}^{5}\right)$. The centers of the monomers are separated by 12 and 11 dipoles, respectively. For further explanations see Fig. 6.

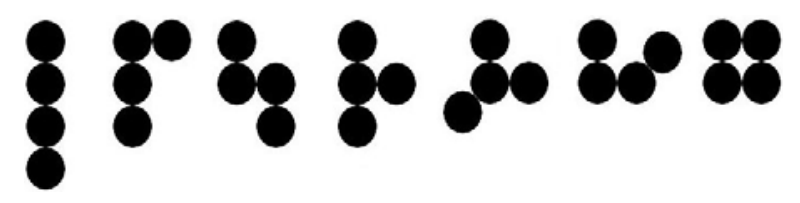

Fig. 8. Different forms for 4 monomers, from left to right: Form 1 to Form 7. All forms are planer except Form 5 and 6 where the monomers at other than a right angle indicate a monomer out of the plane. Form 6 exists in right- and left-handed versions; we assume that their optical properties are equivalent and only include one version.

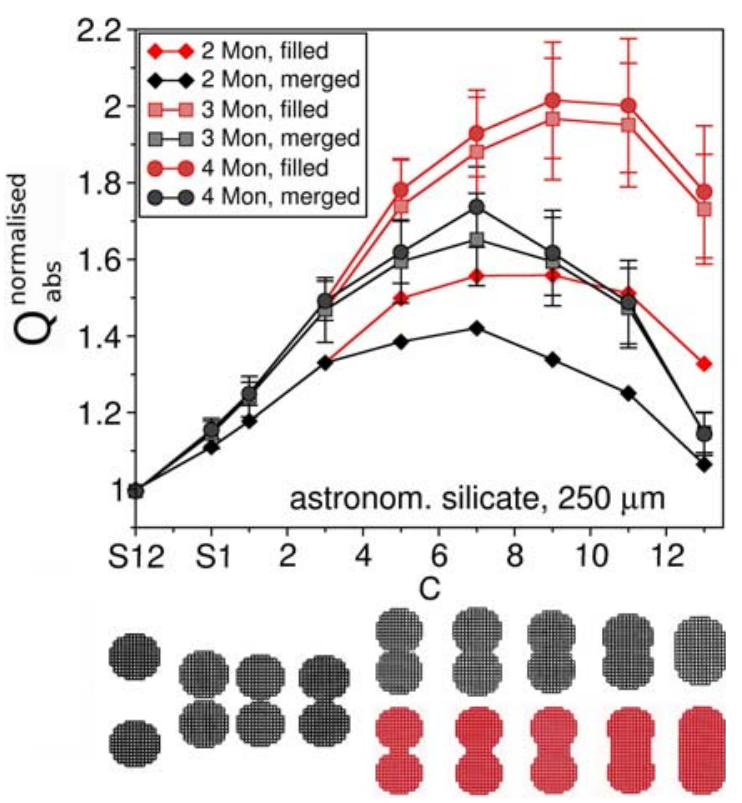

Fig. 9. $Q_{\text {abs }}^{\text {normalised }}$ versus $C$ for aggregates with 2, 3 and 4 monomers with $\varnothing_{13}^{C}$. Results for filled (red curves) and merged (black curves) monomers are shown. The results are averaged over all possible shapes. S12 (S1) indicates the case of monomers separated by 12 (1) dipoles. From left to right on the $x$-axis we follow the contacts presented in Fig. 6 . 


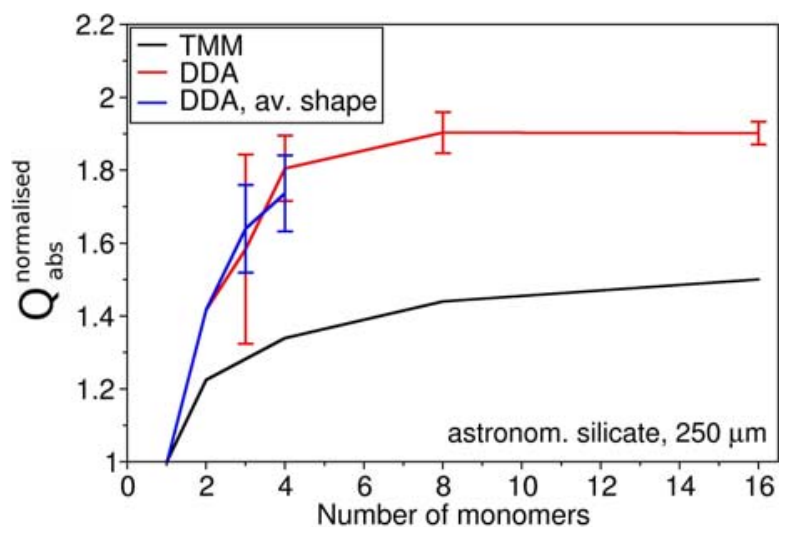

Fig. 10. $Q_{\text {abs }}^{\text {normalised }}$ versus the number of monomers in the aggregate: DDA (red) and with TMM (black). For DDA we consider $\varnothing_{13}^{7}$ and merged monomers.

indicate $C$, the number of dipoles across the contact area. In general, the cases from left to right on the $x$-axis are those shown from left to right in Fig. 6. The black lines correspond to merged monomers (black sketches in Fig. 6) and the red lines correspond to filled monomers (red sketches in Fig. 6). We include the dispersions for the different shapes for aggregates with 3 and 4 monomers. In the case of 2 monomers only one shape is possible and no dispersions occur.

The model calculations show that, with increasing contact area, $Q_{\text {abs }}^{\text {normalised }}$ increases until a maximum is reached, which occurs at $\emptyset_{13}^{7}$ for merged monomers and at $\varnothing_{13}^{9}$ for filled monomers. Repeating these calculations with different numbers of dipoles per monomer, we find in all cases a maximum in $Q_{\text {abs }}^{\text {normalised }}$ at the same ratios of $N / C$.

From these calculations we conclude that $Q_{\text {abs }}$ depends on the contact area between the monomers and that a maximum in $Q_{\mathrm{abs}}^{\text {normalised }}$ is reached when the diameter of the contact area is slightly larger than half the diameter of a monomer. We find the same result for aggregates of amorphous carbon.

\subsubsection{Number of monomers}

To analyse the increase of $Q_{\text {abs }}$ with the number of monomers we calculate $Q_{\mathrm{abs}}^{\text {normalised }}$ for aggregates with $2,3,4,8$ and 16 merged monomers with $\emptyset_{13}^{7}$ which corresponds to the peak in Fig. 9.

The results are shown in Fig. 10 for aggregates of astronomical silicate at a wavelength of $250 \mu \mathrm{m}$. For aggregates with 3, 4, 8 and 16 monomers different shapes of aggregates are possible. We consider a fractal dimension of 2 and average over 10 different shapes (see red curve). For aggregates with a small number of monomers the assumption of a fractal dimension is imprecise and an average over 10 shapes can lead to large deviations (see Jones 2011). Therefore, for aggregates with 3 and 4 monomers we additionally include the equal-weighted averages over all possible shapes (blue curve) (see Sect. 3.2.2). For aggregates of 8 and 16 monomers a large number of shapes are possible which cannot be considered separately. For each case we calculate the dispersions. For aggregates with 2 monomers only one shape of aggregate is possible and there is no dispersion.

We note a large differences in $Q_{\text {abs }}^{\text {normalised }}$ between a single monomer and aggregates of 4 monomers; for larger aggregates $Q_{\text {abs }}$ shows only a weak dependence on the number of monomers.
Figure 10 further shows a comparison with results obtained using TMM (black curve); in general the DDA results are larger due to the effects of contact area. The increase in $Q_{\mathrm{abs}} / a_{\mathrm{V}}$ with increasing number of monomers is steeper for DDA than for TMM.

We conclude that the enhancement in $Q_{\text {abs }}$ calculated with DDA is due to the contact area between the monomers. The enhancement increases with increasing number of monomers and saturates after coagulation of 8 monomers. Similar results are obtained for aggregates of amorphous carbon.

\section{Discussion}

Our results show that the increase in emissivity for aggregates calculated using DDA is the result of larger contact areas between the monomers, compared to TMM and GMM. While for TMM and GMM the connection between the monomers is a point, in DDA the monomers connect by at least one dipole, which is significantly larger. A maximal increase in $Q_{\text {abs }}$ is reached when the contact area is half the diameter of the monomer. From this we conclude that DDA gives good results and that the enhancement in $Q_{\mathrm{abs}}$ occurs due to a physical effect, namely the larger contact area between the monomers. The increase in $Q_{\mathrm{abs}}$ is most pronounced for the coagulation of up to 4 monomers but flattens off with an increased number of monomers. Our results further show that the enhancement in $Q_{\text {abs }}$ with DDA increases with increasing $n$ of the optical constants. Therefore this effect appears stronger at long wavelengths for silicate and increases with increasing wavelength for amorphous carbon. This effect is weak in the UV and visible because $n$ is small at these wavelengths for astronomical silicate and amorphous carbon.

The type of contact area that physically makes the most sense, for a given material aggregate, can be obtained from experimental investigations. A contact area with a length half the diameter of the monomer itself seems to be quite large. In addition, with DDA a continuity of the solid material between the monomers is assumed which might be not the case for real cosmic particles. But a point contact between two monomers is even less likely. In all our calculations spherical monomers were assumed. Laboratory measurements, however, show that IDPs consist of irregularly shaped dust particles and that the contact areas between the "monomers" can be relatively large.

The contact area also depends on the composition of the monomers. For the coagulation of silicate monomers it is physically unlikely to achieve such a large contact areas. For interplanetary dust particle structures the contact areas do appear to be significant. Grains consisting of amorphous carbon might show a larger contact area when they collide with other grains of amorphous carbon because the material is of lower density and inherently "softer". But in this case the enhancement of the absorption coefficient is not as large as for astronomical silicate since $n$ is smaller for amorphous carbon. Assuming core-mantle particles, e.g. Li \& Greenberg (1997) and Jones et al. (1990), might be a realistic approach to understand the coagulation with large contact areas and additionally large enhancements in $Q_{\text {abs }}$ due to a large $n$ at long wavelengths. The effects of core-mantle structures on our results are shown in Appendix A.

In our calculations astronomical silicate was considered. The main reason for choosing this material was that the optical constants are available over a large wavelength range. But in general astronomical silicate is not a real material and was developed from a fit to the observed data. The real part of the astronomical silicate refractive index, $n$, is larger at long wavelength compared 
to real silicates such as olivine or pyroxene, which have $n$ close to 3 (compared to 3.4 for astronomical silicate) at $250 \mu \mathrm{m}$. Thus, olivine and pyroxene will show slightly smaller enhancements in long-wavelength emissivities. However, $n$ is still large enough to give a significant enhancement in the absorption coefficient for coagulated monomers.

As described in the introduction an increased emissivity has been observed at long wavelengths in the ISM. This increase was explained by several authors as due to the coagulation of small grains into aggregates. We show that this increase can be large when large contact areas between the monomers occur, as seen in the case of IDPs. DDA is more suitable for that purpose than the exact methods GMM or TMM. Further we can now explain why this increase only occurs at long wavelengths. This is due to the fact that dust mainly consists of amorphous carbon and silicate which show large values of $n$ in the FIR.

\section{Concluding remarks}

Our calculations show that enhanced emissivities occurs as $n$ increases. In the case of silicate high $n$ occurs at long wavelengths, for amorphous carbon $n$ increases with increasing wavelength. Since observations of the ISM show an increased dust emissivity at long wavelengths and since silicate and amorphous carbon are assumed to be the major components of the dust, the coagulation of the dust grains appears to be a plausible explanation for this increase.

We further show that coagulation into aggregates leads to an enhanced emissivity when the inter-particle contact area is significant ( $\sim$ the monomer radius). With DDA these calculations can be carried out, but with TMM particles only point contacts are possible and a "realistic" contact area treatment is not possible. To analyse dust emission in the ISM at long wavelengths using aggregates, DDA and a "realistic" contact area between the monomers needs to be taken into account.

The largest effect on the emissivity occurs for a relatively small number of particles in the aggregate (4 monomers) and therefore implies that an emissivity enhancement at long wavelengths should occur "rapidly" in the ISM where coagulation is ongoing and in its early stages.

The emissivity enhancement is not significantly affected by the presence of carbon mantles but the effect is weakened if ice mantles are present before coagulation.
Acknowledgements. We thank the referee for helpful comments. This research acknowledges the support of the French Agence National de la Recherche (ANR) through the program Cold dust (ANR-07-BLAN-0364-01).

\section{References}

Bazell, D., \& Dwek, E. 1990, ApJ, 360, 142

Bernard, J. P., Abergel, A., Ristorcelli, I., et al. 1999, A\&A, 347, 640

Bohren, C., \& Huffman, D. 1983, Absorption and Scattering of Light by Small Particles (New York - Chichester - Brisbane - Toronto - Singapore: Wiley and Sons)

Bot, C., Helou, G., Boulanger, F., et al. 2009, ApJ, 695, 469

Boulanger, F., Abergel, A., Bernard, J., et al. 1996, A\&A, 312, 256

Brownlee, D. E. 1978, in Protostars and Planets, ed. T. Gehrels, IAU Colloq., 52, 134

del Burgo, C., \& Laureijs, R. J. 2005, MNRAS, 360, 901

del Burgo, C., Laureijs, R. J., Ábrahám, P., \& Kiss, C. 2003, MNRAS, 346, 403 Draine, B. 1988, ApJ, 333, 848

Draine, B. T., \& Flatau, P. J. 2010, User Guide for the Discrete Dipole Approximation Code DDSCAT 7.1, [arXiv: 1002 .1505]

Draine, B. T., \& Lee, H. M. 1984, ApJ, 285, 89

Fogel, M. E., \& Leung, C. M. 1998, ApJ, 501, 175

Greenberg, J. M., \& Li, A. 1997, Adv. Space Res., 19, 981

Guillet, V., Pineau Des Forêts, G., \& Jones, A. P. 2007, A\&A, 476, 263

Henning, T., Michel, B., \& Stognienko, R. 1995, Planet. Space Sci., 43, 1333

Jones, A. P. 1988, MNRAS, 234, 209

Jones, A. P. 2011, A\&A, 528, A98

Jones, A. P., Duley, W. W., \& Williams, D. A. 1990, QJRAS, 31, 567

Kiss, C., Ábrahám, P., Laureijs, R. J., Moór, A., \& Birkmann, S. M. 2006, MNRAS, 373, 1213

Lehtinen, K., Juvela, M., Mattila, K., Lemke, D., \& Russeil, D. 2007, A\&A, 466, 969

Li, A., \& Greenberg, J. M. 1997, A\&A, 323, 566

Li, A., \& Greenberg, J. M. 1998, A\&A, 331, 291

Mackowski, D. W., \& Mishchenko, M. I. 1996, J. Opt. Soc. Am. A, 13, 2266

Michel, B., Henning, T., Stognienko, R., \& Rouleau, F. 1996, ApJ, 468, 834

Mie, G. 1908, Ann. Phys., 330, 377

Paradis, D., Bernard, J., \& Mény, C. 2009, A\&A, 506, 745

Purcell, E. M., \& Pennypacker, C. R. 1973, ApJ, 186, 705

Ridderstad, M., Juvela, M., Lehtinen, K., Lemke, D., \& Liljeström, T. 2006, A\&A, 451, 961

Rietmeijer, F. 1998, in Materials, Reviews in Mineralogy, ed. J. Papike, (Washington, DC: The Mineralogical Society of America), 36, 2-1

Rouleau, F., \& Matin, P. 1991, ApJ, 377, 526

Rouleau, F., \& Martin, P. G. 1993, ApJ, 416, 707

Stepnik, B., Abergel, A., Bernard, J., et al. 2003, A\&A, 398, 551

Stognienko, R., Henning, T., \& Ossenkopf, V. 1995, A\&A, 296, 797

Warren, S. G. 1984, Appl. Opt., 23, 1206

Wright, E. L. 1987, ApJ, 320, 818

Xu, Y. 1995, Appl. Opt., 34, 4573 


\section{Appendix A: Coated monomers}

We calculate $Q_{\mathrm{abs}}$ for core-mantle particles where we assume that the core consists of astronomical silicate and the mantle consists of either amorphous carbon or water ice. We also calculate $Q_{\mathrm{abs}}$ for particles consisting of only astronomical silicate. The absorption coefficient is calculated with DDA, where we use monomers with $\varnothing_{51}^{\mathrm{C}}$. We consider $N=51$ for all monomers (mantled and bare) so as to exclude any deviations due to surface effects. For core-mantle monomers we assume a mantle thickness of 3 dipoles so that the number of dipoles across the core is $N=45$. A smaller mantle thickness gives divergent results due to the limitations of DDA. The radius of the core is assumed to $0.1 \mu \mathrm{m}$. A core-mantle particle has a radius of $0.113 \mu \mathrm{m}$ so that the mantle has a thickness of $0.013 \mu \mathrm{m}$. For an amorphouscarbon mantle this thickness is larger than $3 \mathrm{~nm}$ assumed by Jones et al. (1990) and for water-ice mantles it is slightly smaller than $14.7 \mathrm{~nm}$ assumed by Guillet et al. (2007). For aggregates we calculate the radius of a volume-equivalent sphere to $0.126 \mu \mathrm{m}$ for the bare cores and $0.143 \mu \mathrm{m}$ for the core-mantle monomers.

We consider the following aggregates: a) a single core, b) a single core surrounded by a mantle, c) two cores separated by one dipole (approximating the TMM case), d) two coremantle particles separated by one dipole (approximating the TMM case), e) two connected cores $\emptyset_{51}^{11}$, f) two connected coremantle particles $\emptyset_{51}^{11}$. All of these cases are illustrated in Fig. A.1.

We calculate the absorption cross section $C_{\text {abs }}$ of the particle and divide by the total volume $V_{\text {core }}$ of the cores. We then normalize to $C_{\mathrm{abs}}^{\mathrm{sph}} / V_{\mathrm{core}}^{\mathrm{sph}}$, where $C_{\mathrm{abs}}^{\mathrm{sph}}$ is either determined for the core or for a core-mantle monomer. $V_{\text {core }}^{\text {sph }}$ is the volume of a core.

The results for $Q_{\mathrm{abs}}^{\text {normalised }}$ are summarised in Tables A.1 and A.2. Table A.1 shows the increase in $Q_{\text {abs }}^{\text {normalised }}$ due to the accretion of a mantle onto a core. The results are normalized to $C_{\mathrm{abs}}^{\mathrm{sph}} / V_{\mathrm{core}}^{\mathrm{sph}}$ for a core. We assume that the mantle is "free" material accreted from that gas that therefore leads to a change in the particles optical propteries. For a mantle of amorphous carbon the increase in $C_{\mathrm{abs}} / V$ is a factor of 1.86 while for water ice the increase is a factor of 1.31. Clearly, adding a carbon mantle for "free" leads to a large increase in the particle emissivity.

In Table A. 2 we show the increase in $Q_{\mathrm{abs}}^{\text {normalised }}$ due to coagulation effects for aggregates of core-mantle monomers (upper case) and pure cores (lower case).

We showed before that a single grain shows the same results as two well-separated monomers. Approaching two monomers (which is comparable with TMM calculations) results in an increase of 1.2 for grains with a mantle of amorphous carbon and for grains consisting of pure astronomical silicate.
Table A.1. Absorption cross section divided by the volume of the core $C_{\text {abs }} / V_{\text {core }}$ normalized to $C_{\text {abs }} / V_{\text {core }}$ of a single astronomical-silicate core.

\begin{tabular}{ccc}
\hline \hline a) & b) & \\
Sphere & CM Sphere & Mantle mat. \\
\hline & & \\
\hline 1.0 & 1.86 & am. carbon \\
1.0 & 1.31 & water ice \\
\hline
\end{tabular}

Notes. For the mantle material we assume amorphous carbon and water ice. The radius of the single core is $0.1 \mu \mathrm{m}$ and the radius of the coremantle particle is $0.113 \mu \mathrm{m}$.

Table A.2. Absorption cross section divided by the volume of the core $C_{\text {abs }} / V_{\text {core }}$ normalized to $C_{\text {abs }} / V_{\text {core }}$ of a single core-mantle grain (upper) or core of astronomical silicate (lower).

\begin{tabular}{|c|c|c|c|}
\hline b) & d) & f) & \\
\hline CM Sphere & Not con. CM spheres & Con. CM sphere & Mantle mat. \\
\hline (2) & (2) & 00 & \\
\hline 1.0 & 1.20 & 1.33 & am. carbon \\
\hline 1.0 & 1.13 & 1.17 & water ice \\
\hline a) & c) & e) & \\
\hline Sphere & Not con. spheres & Con. sphere & \\
\hline 1.0 & 1.20 & 1.36 & bare cores \\
\hline
\end{tabular}

Notes. The mantle materials are amorphous carbon and water ice. We also show the results for pure astronomical-silicate grains. The radius of the single core-mantle grain is $0.113 \mu \mathrm{m}$ and the volumeequivalent radius of an aggregate consisting of core-mantle monomers is $0.143 \mu \mathrm{m}$. In the case of pure astronomical-silicate grains, a single grain is $0.1 \mu \mathrm{m}$ in radius while the volume-equivalent radius of the aggregate is $0.126 \mu \mathrm{m}$.

Aggregates with a mantle of water ice show only an increase of 1.13. In the case of connected monomers the increase is largest for astronomical-silicate cores with 1.36. Astronomical silicate shows the largest $n$ at $\lambda=250 \mu \mathrm{m}$ compared to the other materials. The increase assuming a mantle of amorphous carbon is also large with 1.33. With a mantle of water ice the increase is only by a factor of 1.17. Essentially, the accretion of carbon mantles before accretion will not affect the enhanced emissivity. However, if ice mantle accrete before coagulation the "available" enhancement in emissivity is reduced by about a factor of 2 . 
M. Köhler et al.: Aggregate dust connections and emissivity enhancements

a) b)

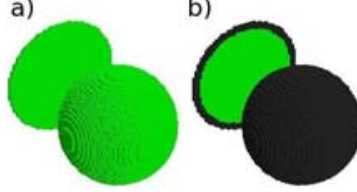

c)

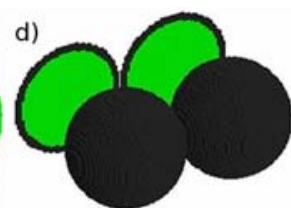

e)

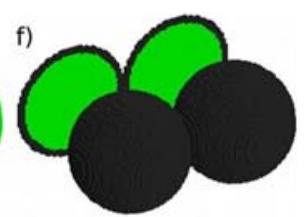

Fig. A.1. Core-mantle particles constructed of dipoles together with their projections are shown. Each dipole is represented by one cube, green dipoles indicate astronomical silicate and black dipoles indicate amorphous carbon. $\varnothing_{51}^{C}$. The projection shows the distribution of dipoles with different materials in the centre of the aggregate. a) single sphere of core material, b) a single core-mantle particle, c) two separated spheres of core material, d) two separated core-mantle particles, e) two connected spheres of core material, $\varnothing_{51}^{11}$, f) two core-mantle monomers connected, $\emptyset_{51}^{11}$.

A96, page 9 of 9 